Operating point selection for dendritic growth during rapid solidification

This content has been downloaded from IOPscience. Please scroll down to see the full text. 2012 IOP Conf. Ser.: Mater. Sci. Eng. 27012076

(http://iopscience.iop.org/1757-899X/27/1/012076)

View the table of contents for this issue, or go to the journal homepage for more

Download details:

IP Address: 129.11.76.232

This content was downloaded on 29/07/2014 at 13:43

Please note that terms and conditions apply. 


\title{
Operating point selection for dendritic growth during rapid solidification
}

\author{
A M Mullis \\ Institute for Materials Research, University of Leeds, Leeds LS2-9JT, UK.
}

\begin{abstract}
A phase-field model of non-isothermal solidification in binary alloys has been used to study the variation of growth velocity, dendrite tip radius and radius selection parameter, $\sigma^{*}$, as a function various process and materials parameters. We find that $\sigma^{*}$ is non-constant and, over a wide parameter space, displays first a local minimum followed by a local maximum as the undercooling is increased. This behaviour is contrasted with a similar type of behaviour to that predicted by simple marginal stability models to occur in the radius of curvature for constant $\sigma^{*}$.
\end{abstract}

\section{Introduction}

As dendrites are self-similar when scaled against the tip radius, $\rho$, the ability to predict $\rho$ is a problem of central importance to dendritic growth theory. However, the prediction of $\rho$ has proved exceptionally challenging. Early analytical solutions [1] predicted that it was the dimensionless Peclet number, $P t=V r / 2 \alpha,(V=$ growth velocity, $\alpha=$ thermal diffusivity $)$, that was related to undercooling, $\Delta T$ leading to a degeneracy in the product $V r$ not observed in nature and thus sparking the search for an additional mechanism to set the length scale, $\rho$, for the dendrite. One of the most enduring solutions to this problem is that of marginal stability [2], which postulates that the dendrite will grow at the largest value of $\rho$ which is stable against the growth of small perturbations. The principal prediction of this theory is that capillary forces break the degeneracy via the relationship

$$
\rho^{2} V=\frac{2 \alpha d_{\mathrm{o}}}{\sigma^{*}}
$$

where $d_{\mathrm{o}}$ is a capillary length and $\sigma^{*}=1 /\left(4 \pi^{2}\right) \approx 0.0253$ [2] is the so-called stability constant.

This theory, particularly in its more sophisticated forms due to Lipton, Glicksman \& Kurz (LGK) [3] and Lipton, Kurz \& Trivedi (LKT) [4], was reasonably successful, with direct simultaneous measurement of $V$ and $\rho$ [5] yielding an experimental value for $\sigma^{*}$ of 0.0195 , in close agreement with theory. However, boundary integral methods established that it is crystalline anisotropy [6] rather than stability per se that is responsible for breaking this degeneracy. The analysis reveals that in the limit of vanishing $P t$ an equation similar to that arising from stability arguments is recovered, but with a radius selection parameter, $\sigma^{*}$, that varies as $\varepsilon^{7 / 4}$, where $\varepsilon$ is the anisotropy strength.

Further progress has been made towards understanding solidification phenomena by the advent of by phase-field modelling, particularly through the formulation of the quantitatively valid 'thin interface model' [7], from which $\sigma^{*}$ may be calculated. Such calculations have shown that for both the thermally controlled solidification of a pure substance [7] and the isothermal solidification of alloys [8], $\sigma^{*}$ appears to decreases linearly with increasing Peclet number. Moreover, in the limited number 
of cases where phase-field models have been applied to alloy systems solidifying under coupled thermo-solutal control $[8,9,10,11], \sigma^{*}$ has been found to vary non-monotonically with both concentration and Peclet number, a result that appears to be borne out experimentally [12]

This potentially makes the estimation of $\sigma^{*}$ extremely problematic. Moreover, as pointed out by Rebow \& Browne [13], the stability constant is an intrinsic part of many alloy solidification models, including cellular automaton, front-tracking and one-domain multiphase models. Such models have tended to use (either explicitly or implicitly) the marginal stability value of $\sigma^{*}$, although other values, either calculated or experimentally estimated [14], can be used, permitting the model to be tuned to the specific material and growth conditions system being simulated. However, the complex, nonmonotonic variation observed in $\sigma^{*}$ for alloy systems solidifying far from equilibrium, means that estimating the value of $\sigma^{*}$ without computationally expensive calculations is not possible.

In this paper we use a phase-field model of coupled thermo-solutal solidification to explore the variation of $\sigma^{*}$ over a range of processing and materials parameters.

\section{Description of the model}

The model adopted here is based upon that of [9] in which, following non-dimensionalization against characteristic length and time scales, $W_{0}$ and $\tau_{0}$, the evolution of the phase-field, $\phi$, and the dimensionless concentration, and temperature fields $U$, and $\theta$ are given by

$$
\begin{gathered}
A^{2}(\psi)\left[\frac{1}{L e}+M c_{\infty}\left[1+\left(1-k_{E}\right) U\right]\right] \frac{\partial \phi}{\partial t}=\nabla \cdot\left(A^{2}(\psi) \nabla \phi\right)+\phi\left(1-\phi^{2}\right)-\lambda\left(1-\phi^{2}\right)^{2}\left(\theta+M c_{\infty} U\right) \\
-\frac{\partial}{\partial x}\left(A(\psi) A^{\prime}(\psi) \frac{\partial \phi}{\partial y}\right)+\frac{\partial}{\partial y}\left(A(\psi) A^{\prime}(\psi) \frac{\partial \phi}{\partial x}\right) \\
\left(\frac{1+k_{E}}{2}-\frac{1-k_{E}}{2} \phi\right) \frac{\partial U}{\partial t}=\nabla \cdot\left(D \frac{1-\phi}{2} \nabla U+\frac{1}{2 \sqrt{2}}\left|1+\left(1-k_{E}\right) U\right| \frac{\partial \phi}{\partial t} \frac{\nabla \phi}{|\nabla \phi|}\right)+\frac{1}{2}\left(\left|1+\left(1-k_{E}\right) U\right| \frac{\partial \phi}{\partial t}\right) \\
\frac{\partial \theta}{\partial t}=\alpha \nabla^{2} \theta+\frac{1}{2} \frac{\partial \phi}{\partial t}
\end{gathered}
$$

where, for 4-fold growth, $A(\psi)=1+\varepsilon \cdot \cos (4 \psi), \psi$ being the angle of the outward pointing normal to the solid-liquid interface, $L$ and $c_{p}$ are the latent and specific heats respectively, and $\lambda$ is a coupling parameter given by $\lambda=D / a_{2}=a_{1} W_{0} / d_{0}$ with $a_{1}$ and $a_{2}$ taking the values $5 \sqrt{ } 2 / 8$ and 0.6267 respectively [7]. $U, \theta$, and $M$ are related to the physical concentration, $c$, temperature, $T$, and liquidus slope, $m$, via

$$
U=\frac{1}{1-k_{E}}\left(\left(\frac{2 c / c_{\infty}}{1+k_{E}-\left(1-k_{E}\right) \phi}\right)-1\right), \theta=\frac{c_{p}\left(\Delta T-m c_{\infty}\right)}{L} \text { and } M=\frac{|m| c_{p}\left(1-k_{E}\right)}{L}
$$

The equations are discritized utilising a quadrilateral finite difference mesh with adaptive local refinement. Second order central difference stencils are used for the calculation of differentials while, to reduce the mesh induced [15] anisotropy, a compact 9-point scheme is used for Laplacian terms.

It has been shown elsewhere that if an explicit temporal discretization scheme is used for this problem the maximum stable time-step is given by $\Delta t \leq C h^{2}$, where $h$ is the minimum mesh spacing and $C=C(\lambda, L e, \Delta T)$, with $C$ varying from $\approx 0.3$ at $L e=1$ to $C \leq 0.001$ at $L e=500$ [16], leading to unfeasibly small time-steps at high Lewis number. Consequently, an implicit temporal discretization is employed here based on the second order Backward Difference Formula with variable time-step.

When using implicit time-stepping it is necessary to solve a large sparse system of algebraic equations at each time-step. Multigrid methods are among the fastest available solvers for such 
systems and here we apply the non-linear generalization known as FAS [17], with a fully-coupled, nonlinear weighted Gauss-Seidel iteration. Local adaptivity is accommodated via the multilevel algorithm proposed in [18]. Full details of the numerical scheme are given in [10, 16].

We obtain from the model $V$ and $\rho$, the latter being obtain by fitting a parabolic profile to the $\phi=0$ isoline using a $4^{\text {th }}$ order interpolation scheme [10]. We also calculate the equivalent radius selection parameter, $\sigma^{*}$. Following [8], and so as not to make recourse to the Ivantsov function, we use

$$
\sigma^{*}=\frac{d_{0}}{\rho}\left[\xi_{\mathrm{T}} P t+2 \xi_{c} P c \frac{|m| c_{\infty}}{L / c_{p}}\left(\frac{1-k_{E}}{1-\left(1-k_{E}\right) \Delta c}\right)\right]^{-1}
$$

where $\Delta c$ is the local concentration 'frozen in' at the interface (taken as $\phi=0$ ). The value of $\sigma^{*}$ thus obtained may be compared with that of the LGK [3] stability model by setting $\xi_{\mathrm{T}}=\xi_{\mathrm{c}}=1$ or to the LKT model [4], which is the approach taken here, by applying the high undercoolings corrections

$$
\xi_{\mathrm{T}}=1-\left(1+\frac{1}{\sigma^{*} P t^{2}}\right)^{-\frac{1}{2}}, \xi_{c}=1+2 k_{E}\left(1-2 k_{E}-\left(1+\frac{1}{\sigma^{*} P c^{2}}\right)^{\frac{1}{2}}\right)^{-1}
$$

The convergence behaviour of the model has been studied by [8] for an explicit solver and by [10] for the implicit multigrid solver. In both cases the tests were conducted in the rapid solidification regime $(\Delta=0.55)$. [8] expressed concerns about convergence as they found that $V d_{0} / \alpha$ systematically increased by around $25 \%$ as $\lambda$ was increased from 1 to 4 . Conversely [10] found that when using an implicit multigrid solver $V d_{0} / \alpha$ remained within $\pm 4 \%$ of its mean value when $\lambda$ was varied in this way

\section{Results}

All simulations were run on a $[-1600: 1600]^{2}$ domain with a minimum grid spacing of $h=0.78$, equivalent to a uniform mesh size of $2^{12} \times 2^{12}$. Our baseline parameter set is given by $L e=200$, $k_{E}=0.3, M c_{*}=0.05, \lambda=1$, and $\varepsilon=0.02$, with the Lewis number, $L e$ and alloy concentration (via $M c_{*}$ ) being varied in turn (all other parameters being held constant). The undercooling range investigated in each case is $\Delta=0.2-0.8$. The Lewis number has been varied over the range $L e=50-500$ by varying $\alpha$, with $D$ being kept constant (in order to keep $\lambda$ constant via the relationship by $\lambda=D / a_{2}=a_{1} W_{0} / d_{0}$, which in turn sets the width of the diffuse interface), while $M c_{*}$ has been varied in the range 0.02-0.10.

In the simulations presented here it is the equilibrium value of the partition coefficient that is used as an input parameter, although as discussed elsewhere [19] in the coupled, thermo-solutal model, even when an anti-trapping current is included in the formulation of the problem, some level of solute trapping is observed, giving rise to a velocity dependant partition coefficient. The extent of the observed solute trapping depends upon the width of the diffuse interface, $W_{0}\left(=\lambda d_{0} / a_{1}\right)$ and, as shown in [19], is of a comparable size to that observed experimentally if $W_{0}$ is chosen to be realistic, i.e. to be of the order of 2-3 atom widths, which is the length scale over which long-range crystal order is lost at the solid-liquid interface of a continuous (dendritic) crystal. By taking $\lambda=1$ here we have $W_{0}=1.13 d_{0}$, which with $d_{0}$ typically of the order $0.2-0.5 \mathrm{~nm}$ satisfies this condition.

In all cases the velocity (not shown) increases monotonically with undercooling, displaying a power law dependence, $V \propto \Delta^{\beta}$, with the most dilute alloys and those at highest Le growing most rapidly. This behaviour is much as we would expect, both from consideration of the analytical Ivantsov solutions and from experimental data $[20,21,22]$ on 3-dimensional free dendritic growth.

The calculated tip radius, as determined from the phase-field model, is given in figures $1 \mathrm{a} \& \mathrm{~b}$ for variation of $L e$ and $M c_{*}$ respectively. These may be compared (figure $2 \mathrm{a} \& \mathrm{~b}$ respectively) with that which would be expected from the (2-dimensional) LKT [4] model with the same input parameter set (and constant $\sigma^{*}$ ). In all cases the tip radius, as determined from the phase-field model, passes through 
a local minimum as the undercooling is increased. This minimum moves systematically to lower undercooling as $L e$ is increased, although at low $L e$ it is very poorly developed. Also as expected, at low undercooling increasing the alloy concentration generally results in a sharpening of the tip. However, LKT theory predicts that, in addition to a local minimum the tip radius should also display a local maximum at yet higher undercooling, after which the tip radius declines steadily with undercooling. This behaviour is not observed in any of the phase-field simulations.
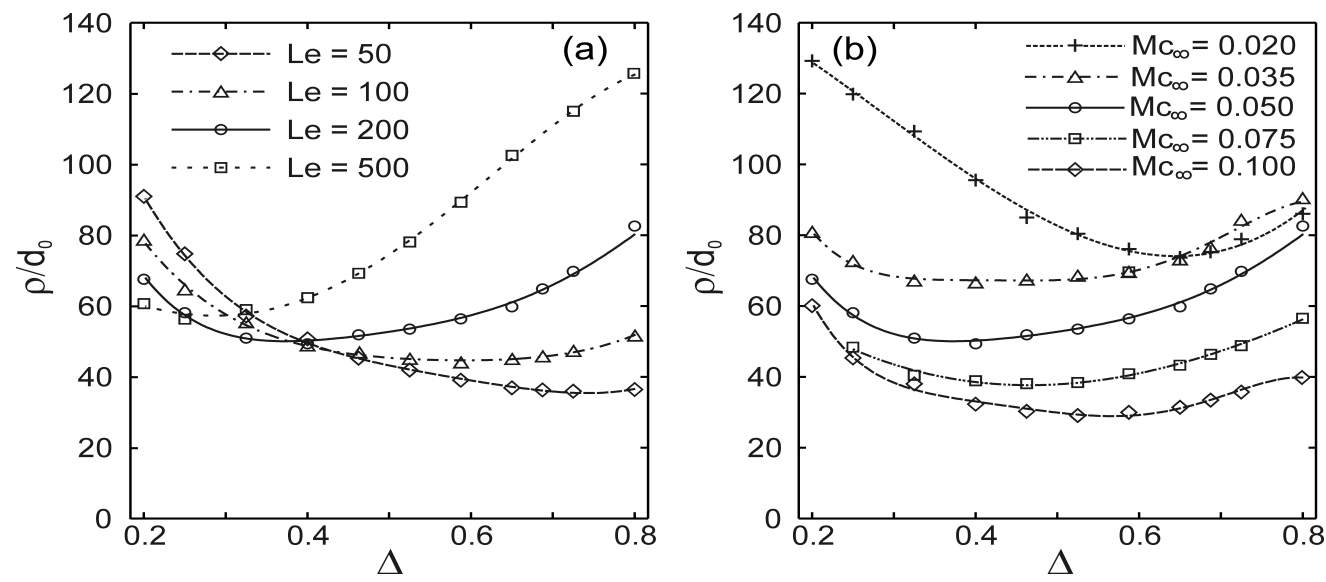

Figure 1. Radius of curvature at the dendrite tip as a function of undercooling, calculated by the phase-field model, for dendrites growing at varying (a) Lewis number and (b) concentration.
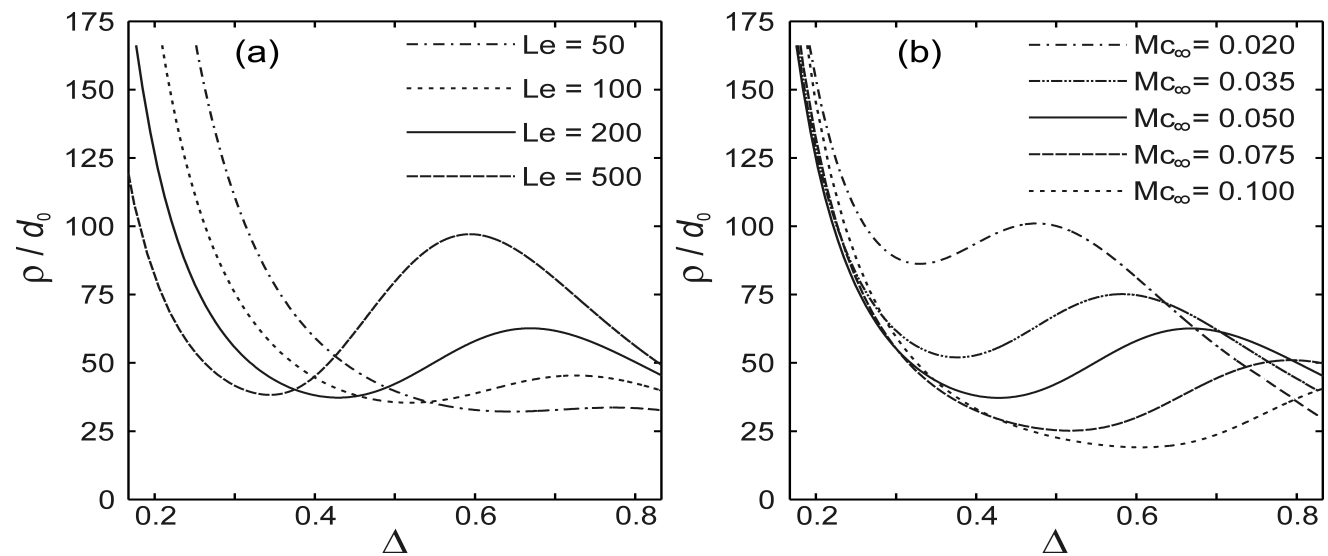

Figure 2. Radius of curvature at the dendrite tip as a function of undercooling as predicted by LKT theory for variation of (a) $L e$ and (b) $M c_{x}$, on the assumption of constant stability parameter, $\sigma^{*}$. Parameters are the same as used in the phase-field model

The dependence of the dendrite tip radius upon undercooling, as predicted by LKT theory, has very much been a cornerstone of rapid solidification theory. However, experimental evidence in support of such a local minimum or maximum is scant. Transparent analogue casting alloys can only be undercooled by very small amounts, so that the appropriate undercooling range is not accessible. In metallic systems only an indirect estimate of the tip radius is possible, generally by assuming that the grain size or dendrite trunk radius [23] scales as a constant multiple of the tip radius. However, although there is plentiful evidence of an initial decrease in microstructural length scale, it has proved almost impossible to make a continuous extension of this analysis to high undercoolings where the presence of a local minima/maxima might be inferred. Even for systems where a single dendritic phase exists over the whole undercooling range, such as $\mathrm{Ni}-\mathrm{Cu}$ [20] or $\mathrm{Cu}-\mathrm{O}$ [24], remelting and/or recrystallisation effects such as spontaneous grain refinement $[23,25,26]$ make the estimation of the 
original tip radius impossible. Given that the local minimum in $\rho$ moves to lower undercooling as $L e$ is increased, we consider it likely that at higher Lewis numbers than those studied a local maximum in $\rho$ may be observed. However, from the results presented here we conclude that the occurrence of such maxima is nowhere near as ubiquitous as suggested by marginal stability models.

The value of $\sigma^{*}$ estimated from the phase-field model is shown in figures $3 \mathrm{a} \& \mathrm{~b}$ (again for variation of $L e$ and $M c$ r respectively). In all cases, except for the most concentrated alloy studied in which the minimum does not occur until $\Delta=0.725, \sigma^{*}$ shows first a local minimum, followed by a local maximum, as the undercooling is increased. The location of both this minima and maxima shift to higher undercooling as either the concentration is increased or the Lewis number decreased. Moreover, the amplitude of the variation also increases with $L e$. In fact qualitatively, considering only the general shapes of the curves, there is a far greater similarity between the marginal stability curve for the radius and the dependence predicted here for the stability parameter, $\sigma^{*}$, than there is between the marginal stability curve and the actual tip radius predicted by the phase-field model.
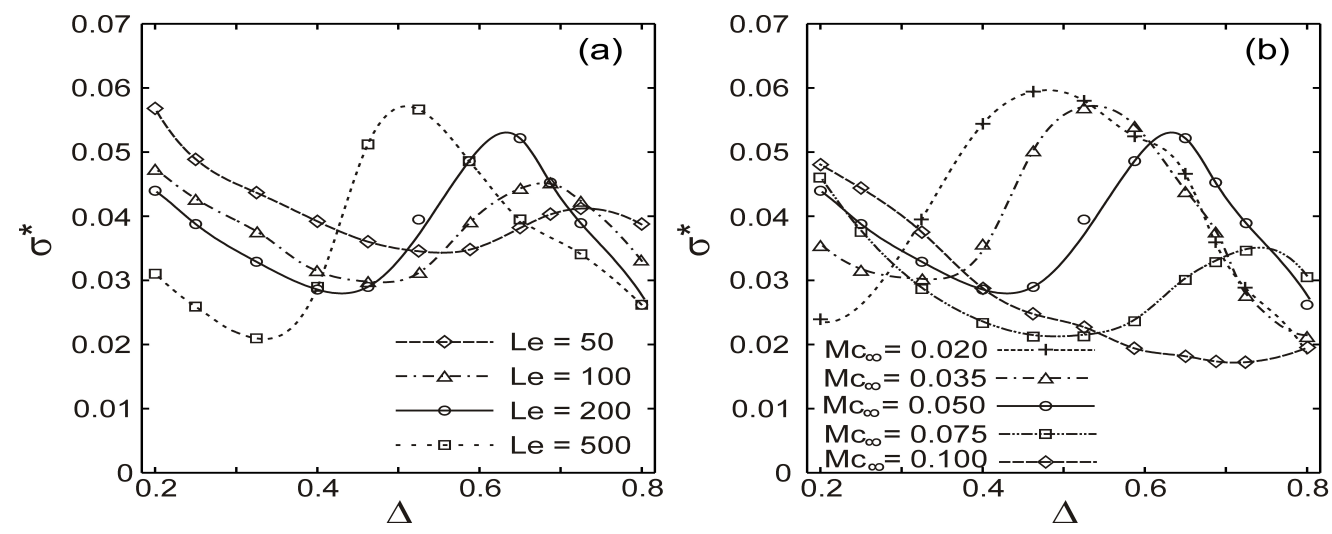

Figure 3. Values of the stability parameter, $\sigma^{*}$, as a function of undercooling, as calculated by the phase-field model, for dendrites growing at varying Lewis number and varying (a) Lewis number and (b) concentration.

\section{Discussion}

A phase-field model of coupled thermo-solutal growth, formulated in the thin-interface limit, has been used to calculate the radius of curvature at the tip of a dendrite growing into its undercooled parent melt over a wide parameter space. We find that although all of our radius curves display a local minimum as the undercooling is increased, none display a local maximum. The idea of the local minimum followed by local maximum in the dendrite tip radius has become common currency within the rapid solidification community. This work would suggest possibly mistakenly so.

Moreover, if we make a qualitative comparison, many of the features which are characteristic of marginal stability predictions for the tip radius (i.e. local minimum followed by local maximum), are reproduced by the phase-field model in its prediction of $\sigma^{*}$. We draw this analogy not because we ascribe any particular validity to the ideas of marginal stability, we do not, but because in the case of an analytical model it is easier to understand where affects may arise in a way that is not always the case in a numerical model. Thus the analogy is to aid the interpretation of the phase-field results and to attempt to elicit physical understanding.

We first consider the simpler case of growth with a single diffusing species, either heat or solute. Marginal stability theory predicts a monotonically decreasing tip radius as the undercooling (or equivalently Peclet number) is increased. Conversely, both phase-field and microscopic solvability theory predict that in this case $\sigma^{*}$ will decrease monotonically. In the LKT model it is well understood how two similar curves, each showing a monotonic decrease, but controlled by different species diffusing over very different length and time scales, can give rise to the characteristic alloy curve (e.g. figure $2 \mathrm{a} \& \mathrm{~b}$ ). The initial low undercooling region is the result of the dendrite growing under solute 
control, with $\rho$ decreasing as the solutal Peclet number increases. At intermediate undercoolings we have high solutal but low thermal Peclet numbers and growth control begins to be transferred to thermal diffusion, with a commensurate increase in $\rho$. Finally, at high undercooling thermal diffusion dominates the growth process. The radius increase that was observed as control moves to thermal diffusion is reversed, leading to a local maximum in the radius followed by a steady decline as growth moves into the high thermal Peclet number regime.

Of course, all of this assumes constant $\sigma^{*}$. If we now consider the case in which $\sigma^{*}$ is not constant we may apply a similar argument, but now to the selection parameter, $\sigma^{*}$, rather than the tip radius per se. Consequently, as the undercooling is increased it will be $\sigma^{*}$ that shows the characteristic local minimum followed by local maximum. If we then accept that it is $\sigma^{*}$ and not the tip radius directly that is determined by the competition between thermal and solutal transport processes, we can begin to understand why the calculated tip radius shows the complex behaviour observed. In particular, the argument above, and the results presented here, suggests that at sufficiently high undercooling the behaviour of $\sigma^{*}$ will be to decrease with increasing undercooling. As low $\sigma^{*}$ may be associated with large tip radius, as described by equation (1), it follows that for a sufficiently rapid decrease in $\sigma^{*}$ the tip radius will continue to increase with increasing undercooling, rather than displaying a local maximum and then decreasing, in much the fashion that is observed in the simulations presented here. That is not to say that this will occur in all cases, indeed, a parameter space may well exist in which the decrease in $\sigma^{*}$ is sufficiently slow to permit the tip radius to also decrease as the undercooling is increased. However, as observed here, that parameter space may not be ubiquitous in the way assumed in marginal stability theory. The above hypothesis is presented as an argument, which, at least qualitatively, allows the results of the phase-field simulation to be rationalised.

\section{References}

[1] Ivantsov G P 1957 Doklady Akademii Nauk SSSR 58567.

[2] Mullins W W and Sekerka R F 1964 J. Appl. Phys. 33444.

[3] Lipton J, Glicksman M E and Kurz W 1984 Mater. Sci.Eng. 6557.

[4] Lipton J, Kurz W and Trivedi R 1987 Acta Metall. 35957.

[5] Huang S C and Glicksman M E 1981 Acta Metall 29701.

[6] Ben-Jacob E, Goldenfeld N, Kotliar B and Langer J 1984 Phys. Rev. Lett. 532110.

[7] Karma A and Rappel W J 1998 Phys. Rev. E 574323.

[8] Ramirez J C and Beckermann C 2005 Acta Mater. 531721.

[9] Ramirez J C, Beckermann C, Karma A and Diepers H -J 2004 Phys. Rev. E 69051607.

[10] Rosam J, Jimack P K and Mullis A M 2008 Acta. Mater. 564559.

[11] Rosam J, Jimack P K and Mullis A M 2009 Phys. Rev. E 79030601.

[12] Li Q and Beckermann C 2002 J. Cryst. Growth 236482.

[13] Rebow M and Browne D J 2007 Scripta Mater. 56481.

[14] Martorano M A and Biscuola V B 2006 Modell Simul Mater. Sci. Eng. 141225.

[15] Mullis A M 2006 Comp. Mater. Sci. 36345.

[16] Rosam J, Jimack P and Mullis A 2007 J. Comp. Phys. 2251271.

[17] Trottenberg U, Oosterlee C and Schuller A, Multigrid (Academic Press 2001).

[18] Brandt A 1977 Math. Comp. 31333.

[19] Mullis A M, Rosam J, Jimack P K 2010 J. Cryst. Growth 3121891.

[20] Willnecker R, Herlach D M and Feuerbacher B 1989 Phys. Rev. Lett. 622707.

[21] Battersby S E, Cochrane R F and Mullis A M 1997 Mater. Sci. Eng. A 226443.

[22] Dragnevski K I, Cochrane R F and Mullis A M 2002 Phys. Rev. Lett. 89215502.

[23] Schwarz M, Karma A, Eckler K and Herlach D M 1994 Phys. Rev. Lett. 731380.

[24] Battersby S E, Cochrane R F and Mullis A M 2000 J. Mater. Sci. 351365.

[25] Mullis A M 1997 Int. J. Heat Mass Transfer 404085.

[26] Dragnevski K, Cochrane R F and Mullis A M 2004 Mater. Sci. Eng. A 375-377 479. 